\title{
The Relationship between Sexual and Emotional Promiscuity and Infidelity
}

\author{
By Ricardo Pinto* \\ Joana Arantes ${ }^{\dagger}$
}

\begin{abstract}
The main aim of the present study is to relate, for the first time, the sexual and emotional sides of infidelity, that is characterized by any form of close physical or emotional involvement with another person while in a committed relationship with promiscuity, which is typically defined by the search for the maximum sexual pleasure or how easily and often someone falls in love. Another aim was to investigate potential sex differences within both domains. For that, 369 participants (92 males and 277 females) answered to an online questionnaire that collected information about infidelity and promiscuity. More specifically, participants were asked to complete the revised Sociosexual Orientation Inventory (SOI-R), the Emotional Promiscuity (EP) Scale, and the Sexual and Emotional Infidelity (SEI) scale. In addition, some sociodemographic questions, as well as history of infidelity questions, were also asked. The analyses included Pearson correlations, ANOVA and t-tests. Results show that all domains are related, specifically sexual and emotional infidelity with sexual and emotional promiscuity.
\end{abstract}

Keywords: Emotional promiscuity, Emotional infidelity, Sex differences, Sexual infidelity, Sexual promiscuity.

\section{Introduction}

In a world where infidelity and promiscuity are increasingly experienced (Brand et al. 2007, Jones and Paulhus 2012), few studies have focused on their emotional and sexual domains. The infidelity and the promiscuity can have an important impact on individuals and on intimate relationships (Silva et al. n.d., Vangelisti and Gerstenberger 2004). For example, the infidelity is one of the most common reasons for divorce and couple therapy (Glass and Wright 1992). In addition, promiscuity is known to have a negative effect on healthy living (Okafor and Duru 2010).

The most accurate definition of infidelity may be the one that states infidelity as any form of involvement, romantic or sexual, short or long-term, while the individual is in a committed romantic relationship with another person (Brand et al. 2007). It is known that for many people, infidelity only incorporates the involvement in unfaithful sexual behaviors, but in fact it can also refer to emotional betray (Brand et al. 2007, Leeker and Carlozzi 2012). Therefore, infidelity can be divided into two domains: sexual infidelity and emotional

\footnotetext{
* Clinical Psychologist, University of Minho, Portugal.

${ }^{\dagger}$ Invited Assistant Professor, University of Minho, Portugal.
} 
infidelity (Kinsey et al. 1949). Sexual infidelity is the occurrence of sexual acts with a third person, violating the ground rules established by the romantic couple, and these acts range from kissing and fondling, to sexual intercourse, including oral, vaginal and anal sex (Brand et al. 2007, Leeker and Carlozzi 2012). On the other hand, emotional infidelity is characterized by the involvement with a third party in emotional acts, where the ground rules established by the couple are broken, and these acts involve falling in love with another person, being vulnerable with another, being more committed to another, flirting, dating, share deep thoughts with another, among other behaviors (Barta and Kiene 2005).

Infidelity problems affect men and women, with both sexes engaging in extra-conjugal behaviors (Atkins et al. 2001). However, men tend to engage more in sexual affairs, whereas women in emotional affairs (Sagarin et al. 2003). Research (Green and Sabini 2006, Shackelford et al. 2002) has also shown that women find it more difficult to deal with emotional infidelity and are more likely to finish a relationship due to this behaviors, whereas men have more difficulty to deal with sexual infidelity and are more prone to terminate a relationship due to sexual infidelity.

Some authors defend that infidelity may come as a consequence of promiscuity, and that frequently both concepts go side by side (Feldman and Cauffman 1999, Mark et al. 2011). Promiscuity can be understood as the willingness to engage in sexual activities with several partners, have casual sex and get involved in sexual activities sooner rather than later (Jones and Paulhus 2012). As opposed to infidelity, there are only few studies in the field of promiscuity (Jones 2011, Jones and Paulhus 2012, Markey and Markey 2007, Penke and Asendorpf 2008, Schmitt 2004). However, promiscuity can also be divided into two domains: sexual and emotional (Jones and Paulhus 2012, Markey and Markey 2007). Sexual promiscuity can be defined as the engagement in uncommitted sexual activities, with non-monogamous partners (i.e. one-night stands), and with multiple partners (Garcia et al. 2010). Emotional promiscuity, on other hand, is the tendency to fall in love easily and often (Jones and Paulhus 2012). This is a recent concept in the literature, with only one manuscript focused on this topic (Jones and Paulhus 2012).

Sociosexuality is a term strongly associated with sexual promiscuity, essentially because sociosexuality describes individual differences in the willingness to one engage in uncommitted sexual relations, where no closeness, commitment, among other indicators of emotional bonding are present (Kinsey et al. 1949). A study on sex differences in the field of sociosexuality showed that men possess more promiscuous behaviors, therefore engaging in unrestricted practices, whereas women tend to show more restricted behaviors (Buss and Barnes 1986). Even though there are no previous studies that relate both domains of infidelity and promiscuity, few studies have investigated the relationship between infidelity and sexual promiscuity (e.g., number of short-term relationships throughout life). For example, Feldman and Cauffman (1999) analyzed a sample of 417 college students and found that individuals that show permissive behaviors, associated with increased number of sexual partners are more prone to engage in infidelity. Similarly, Barta and Kiene (2005) conducted a study with 432 college 
students, 120 of whom mentioned past infidelity behaviors. Their results showed that those who have an unrestricted sociosexual orientation tend to report a sexual motive for being unfaithful. Also, the fact that men tend to exhibit more an unrestricted sociosexual orientation may account, in part, for the greater frequency of infidelity behaviors among males.

\section{The Current Study}

Infidelity and promiscuity are, as stated before, often side by side in the world we live in (Feldman and Cauffman 1999, Mark et al. 2011). There are studies that focused on sexual and/or emotional infidelity (e.g., Brand et al. 2007, Drigotas et al. 1999, Leeker and Carlozzi 2012, Sagarin et al. 2003, Shackelford et al. 2002, Wilson et al. 2011), as well as studies on sexual and/or emotional promiscuity (e.g. Jones 2011, Jones and Paulhus 2012, Markey and Markey 2007, Penke and Asendorpf 2008, Schmitt 2004). However, no prior scientific research has related all these domains - sexual and emotional for promiscuity and infidelity. Analyzing these variables will constitute an innovation in the field, and will have important applications.

Therefore, our study aims to investigate the relationship between sexual and emotional promiscuity with sexual and emotional infidelity. In addition, we intend to analyze possible sex differences. Our hypotheses are:

1. Emotional promiscuity is positively correlated with emotional infidelity;

2. Sexual promiscuity is positively correlated with sexual infidelity;

3. Sexual and emotional infidelity are positively correlated;

4. Sexual and emotional promiscuity are positively correlated;

5. Men tend to be more sexual promiscuous and sexual unfaithful than women;

6. Women tend to be more emotional promiscuous and emotional unfaithful than men.

\section{Method}

\section{Participants}

Our initial sample included a convenience sample of 630 participants. After excluding partially-completed questionnaires $(n=261)$, our final sample comprised 369 participants. From those, 92 were males $(24.93 \%)$ and 277 were females $(75.07 \%)$. The mean age of participants was 23.40 years $(S D=5.47$; range: 18 to 56 years). In terms of nationality, the majority $(n=357 ; 96.75 \%)$ were Portuguese. Regarding sexual orientation, $334(90.51 \%)$ identified themselves as heterosexual, $23(6.23 \%)$ bisexual and $12(3.25 \%)$ homosexual. In terms of relationship status, $246(66.67 \%)$ said that were currently involved in a close relationship. From those, the mean relational satisfaction was 5.75 $(S D=1.20)$. Among those not currently in a relationship $(n=123 ; 33.33 \%), 91$ 
(73.99\%) said that they had been involved in a relationship in the past, whereas $32(26.01 \%)$ participants said that they had never been in a relationship. Participants did not receive monetary compensation, and were recruited through personal and institutional e-mails, and online social networks.

\section{Measures}

\section{$\underline{\text { Sociodemographic Questionnaire }}$}

Participants answered questions regarding their age, sex, nationality, sexual orientation, relationship status and, those who were in a close relationship, were also asked about their relationship satisfaction using a Likert scale from 1 ("not satisfied at all") to 7 ("extremely satisfied"). Those that were not currently in a close relationship were also asked if they had ever been involved in a close relationship.

\section{The Revised Sociosexual Orientation Inventory (SOI-R)}

The SOI-R (Penke and Asendorpf 2008, translated and validated to Portuguese by Pinto and Arantes, n.d. a) was developed to answer to some criticisms to the original SOI scale (Simpson and Gangestad 1991), namely regarding its psychometric values. The SOI-R has as the main purpose to evaluate the sociosexuality or sociosexual orientation, and it is commonly used to assess sexual promiscuity (e.g. Vrangalova and Ong 2014).

This scale comprises 9 items and it is divided in three parts. The first part is composed by 3 items (e.g., "With how many different partners have you had sexual intercourse on one and only one occasion?"), each scoring on a scale that ranges from 0 to 20 people or more. The second part comprehends 3 items (e.g., "Sex without love is OK."), to be rated on a 5-point Likert scale, ranging from 1 ("strongly disagree") to 5 ("strongly agree"). The third part also includes 3 items (e.g., "How often do you have fantasies about having sex with someone you are not in a committed romantic relationship with?"), each scoring on a 5point Likert scale from 1 ("never") to 5 ("nearly every day").

According to Penke and Asendorpf (2008) and Pinto and Arantes (n.d. a) the SOI-R produces three factors, "behavior", "attitude" and "desire", each assessing, respectively, the behavioral (items 1 to 3), the attitudinal (items 4 to 6) and the hidden desires (items 7 to 9) of sociosexuality. In terms of psychometric values, the three-factor model produces a very good fit $(\mathrm{CFI}=.99, \mathrm{NFI}=.98, \mathrm{SRMR}=$ .04 ; Penke and Asendorpf 2008, and CFI $=.99 ; \mathrm{NFI}=.98 ;$ SRMR $=.03$; Pinto and Arantes n.d. a), and demonstrated good internal reliability ( $\alpha=.83$; Penke and Asendorpf 2008, and $\alpha=.88$; Pinto and Arantes, n.d. a).

\section{Emotional Promiscuity (EP) Scale}

The EP scale (Jones 2011 translated and validated to Portuguese by Pinto and Arantes n.d. a) was developed to assess emotional promiscuity, separately 
from romanticism or sexual promiscuity. This scale comprehends 9 items, scored on a 5-point Likert scale (ranging from 1, "strongly disagree", to 5, "strongly agree"), and one additional item regarding the number of people that they have fallen in love with during their life. The scale is composed by two factors, "easily" and "often". These two domains are associated with emotional promiscuity in literature (Jones 2011), indicating the difficulty and the frequency of falling in love that characterize emotional promiscuous people: they fall in love easily (i.e., low difficulty) and often (i.e., high frequency). Items from 1 to 5 loaded on the "easily" factor (e.g., "I fall in love easily."), and items 6 to 10 on the "often" factor (e.g., "I fall in love frequently."). Also, the two-factor model produced a good fit, and showed an acceptable ( $\alpha=.75$; Jones, 2011) or good $(\alpha=.82$; Pinto and Arantes n.d. a) internal reliability.

\section{$\underline{\text { Sexual and Emotional Infidelity (SEI) Scale }}$}

The main scope of the SEI scale, developed by Pinto and Arantes (n.d. b) is to assess both emotional and sexual infidelity. The final version comprises 14 items, 7 regarding emotional infidelity (e.g., "I give more attention and prefer the company of people other than my partner"), and 7 regarding sexual infidelity (e.g., "I have sexual intercourse (vaginal) with people other than my partner"). Each item is scored on a 7-point Likert scale, with $1=$ "It never happens to me" and 7 = "It happens to me often". All the items were randomly presented. The SEI scale is composed by two domains: sexual and emotional. According to Pinto and Arantes (n.d. b), a good fit of the two-factor model was produced $\left(\chi^{2}\right.$ $=416.15 ; \mathrm{CFI}=.92 ; \mathrm{NFI}=.90 ; \mathrm{TLI}=.90 ; \mathrm{SRMR}=0.05)$. In addition, the scale also demonstrates good internal reliability $(\alpha=.93)$.

\section{$\underline{\text { History of Infidelity Questions }}$}

Participants were asked four questions regarding their own infidelity behaviors. More specifically, participants were asked if they have ever been sexual and/or emotional unfaithful in their past relationships, and (for those currently involved in a close relationship) if they have ever been sexual and/or emotional unfaithful to their current partner.

\section{Procedure}

Participants answered first to the demographic questionnaire, followed by the revised Sociosexual Orientation Inventory (SOI-R), the Emotional Promiscuity (EP) scale, the Sexual and Emotional Infidelity (SEI) scale, and the history of infidelity questions, in a counterbalanced order. Participants that were not currently involved in a close relationship and that had never been in a relationship in the past did not respond to the SEI scale and to the history of infidelity questions. The procedure lasted approximately 10-15 minutes. Participants' responses were recorded on an Internet webpage using Qualtrics software, Version 2013 of the Qualtrics Research Suite (www.qualtrics.com). 


\section{Data Analysis}

All the data collected in our study were exported to an Excel spreadsheet. The analyses were then conducted with Statistical Package for Social Sciences (SPSS, v. 21), and included: i) Pearson correlations, to examine the associations between the different variables in our study; ii) $t$-tests to examine sex differences and to determine differences between participants with different pattern to infidelity behaviors; and iii) ANOVA to examine possible differences between the participants sexual orientation regarding promiscuity and infidelity. Before the analyses were run we ensured that all test assumptions were fulfilled. A criterion of $p<.05$ was used for all significance tests.

\section{Results}

\section{Promiscuity and Infidelity: A Correlational Analysis}

In order to analyze the relation between promiscuity and infidelity, Pearson correlations were conducted. Results are shown in Table 1. Age was significantly positively correlated with sexual promiscuity $[r(359)=.257, p<.001]$, and with emotional promiscuity $[r(361)=.141, p<.01]$, showing that older participants tend to be more promiscuous than younger participants. Additionally, age was significantly positively correlated with both sexual infidelity $[r(327)=$ $.238, p<.001]$ and emotional infidelity $[r(327)=.218, p<.001]$, indicating that older participants tend to be more unfaithful than younger participants.

Relationship satisfaction was significantly negatively correlated with sexual promiscuity $[r(241)=-.322, p<.001]$ and emotional promiscuity $[r(241)=-.243$, $p<.001$ ], indicating that participants who perceived their relationship satisfaction as being lower tend to be more promiscuous. Relationship satisfaction was also significantly negatively correlated with sexual and emotional infidelity $[r(241)$ $=-.196, p<.01$, and $r(241)=-.407, p<.001$, respectively] indicating that participants who perceived their relationship satisfaction as being lower tend to be more unfaithful.

Sexual promiscuity was significantly positively correlated with emotional promiscuity $[r(356)=.261, p<.001]$, as well with sexual infidelity $[r(323)=.595$, $p<.001]$ and emotional infidelity $[r(323)=.676, p<.001]$, indicating that sexually promiscuous participants also tend to be emotionally promiscuous, and sexual and emotional unfaithful. Similarly, emotional promiscuity was significantly positively correlated with sexual infidelity $[r(324)=.123, p<.05]$ and emotional infidelity $[r(324)=.319, p<.001]$, suggesting that emotionally promiscuous participants tend to be emotional unfaithful and sexual unfaithful. Additionally, sexual and emotional infidelity were also significantly positively correlated $[r(327)=.716, p<.001]$, showing that sexual unfaithful participants also tend to be emotional unfaithful. 
Table 1. Correlations between Promiscuity and Infidelity Variables

\begin{tabular}{|l|c|c|c|c|}
\hline & $\begin{array}{c}\text { Emotional } \\
\text { Infidelity }\end{array}$ & $\begin{array}{c}\text { Sexual } \\
\text { Infidelity }\end{array}$ & $\begin{array}{c}\text { Emotional } \\
\text { Promiscuity }\end{array}$ & $\begin{array}{c}\text { Sexual } \\
\text { Promiscuity }\end{array}$ \\
\hline Age & $.218^{* * *}$ & $.238^{* * *}$ & $.141^{* *}$ & $.257 * * *$ \\
\hline $\begin{array}{l}\text { Relation } \\
\text { Satisfaction }\end{array}$ & $-.407 * * *$ & $-.196^{* *}$ & $-.243^{* * *}$ & $-.322^{* * *}$ \\
\hline $\begin{array}{l}\text { Sexual } \\
\text { Promiscuity }\end{array}$ & $.676^{* * *}$ & $.595^{* * *}$ & $.261 * * *$ & \\
\hline $\begin{array}{l}\text { Emotional } \\
\text { Promiscuity }\end{array}$ & $.319^{* * *}$ & $.123^{*}$ & & \\
\hline $\begin{array}{l}\text { Sexual } \\
\text { Infidelity }\end{array}$ & $.716^{* * *}$ & & & \\
\hline
\end{tabular}

\section{History of Infidelity}

In the questionnaire there were dichotomous variables regarding the history of infidelity. Therefore we used t-test to analyze if there were differences regarding promiscuity and infidelity between those participants that have been unfaithful in their past relationships or in their current relation and those participants that were faithful. Results showed that participants that reported been sexually unfaithful in the past were more sexually promiscuous $t(58.88)=8.26, p<$ $.001, d=1.11$, emotionally promiscuous $t(66.44)=3.37, p<.001, d=1.67$, sexually unfaithful $t(50.98)=5.64, p<.001, d=-.56$ and emotionally unfaithful $t(56.52)=6.50, p<.001, d=.31$ than those that have been sexually faithful in the past. Additionally, participants who have been emotionally unfaithful in the past were less satisfied with their current relationship, $t(133.42)=-2.30, p<$ $.05, d=-.32$, more sexually promiscuous $t(154.11)=4.09, p<.001, d=1.23$, emotionally promiscuous $t(185.09)=5.84, p<.001, d=1.77$ and more emotionally unfaithful $t(142.98)=5.03, p<.001, d=.44$.

People that were in a relationship and reported being sexually unfaithful to their partners were less satisfied with their current relationship, $t(30.08)=-$ $3.15, p<.05, d=4.40$, more sexually promiscuous $t(36.38)=9.21, p<.001, d$ $=.62$, emotionally promiscuous $t(35.53)=3.46, p<.001, d=1.82$, sexually unfaithful $t(30.70)=5.38, p<.001, d=-.88$ and emotionally unfaithful $t(32.78)=$ $6.79, p<.001, d=.04$. Similarly, people that stated being emotionally unfaithful to their current partner were less satisfied with their relationship, $t(42.16)=-$ $4.70, p<.001, d=4.40$, more sexually promiscuous $t(47.61)=5.92, p<.001$, $d=.67$, more emotionally promiscuous $t(50.94)=6.17, p<.001, d=1.85$, sexually unfaithful $t(38.22)=3.35, p<.05, d=-.81$ and emotionally unfaithful $t(40.42)=6.63, \mathrm{p}<.001, d=.09$.

\section{Promiscuity and Infidelity: Demographic Variables}

\section{$\underline{\text { Sex Differences }}$}

Figure 1 shows the scores for SOI-R and EP for both males and females. Male participants were found to be more sexually promiscuous $(M=3.22 ; S D=.89)$ 
than females $(M=2.11 ; S D=.71), t(131.23)=10.88, p<.001, d=1.38$. However, regarding emotional promiscuity, no significantly differences were found between sexes $\left(M_{\text {males }}=2.82 ; S D_{\text {males }}=.68 ; M_{\text {females }}=2.71 ; S D_{\text {females }}=.66\right)$, $t(148.73)=1.34, p<.182, d=.16$.

Figure 1. Sex Differences for SOI-R and EP

Sex differences for SOI-R and EP

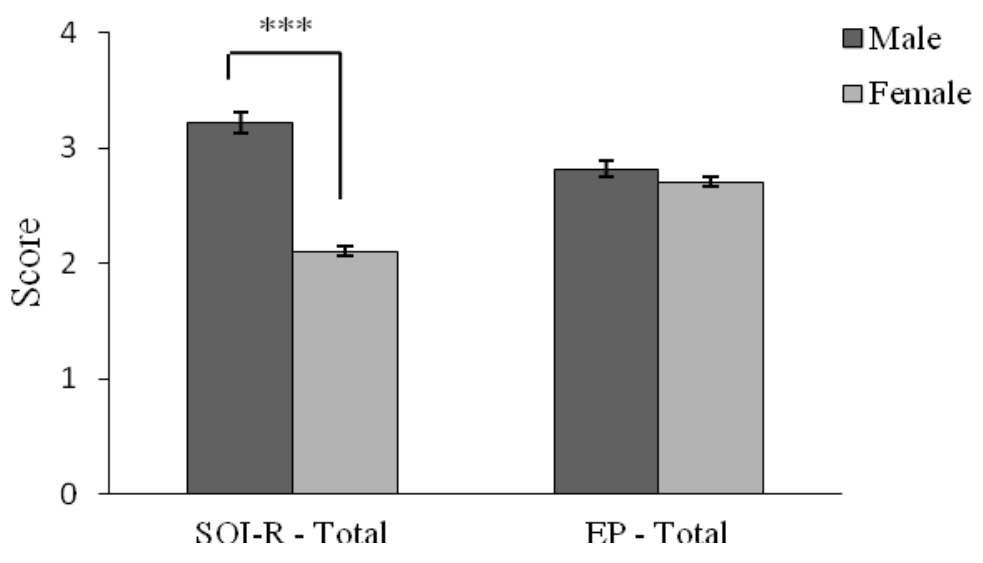

A $t$-test was performed in order to explore if there are any differences between males and females in terms of sexual and emotional infidelity. Results indicated that males are more sexually unfaithful than women $t(86.03)=4.70, p<.001, d$ $=.71$. Additionally, males seem to also be more emotional unfaithful than women $t(99.22)=5.86, p<.001, d=.84$, as shown on Figure 2.

Figure 2. Sex Differences for Sexual and Emotional Infidelity

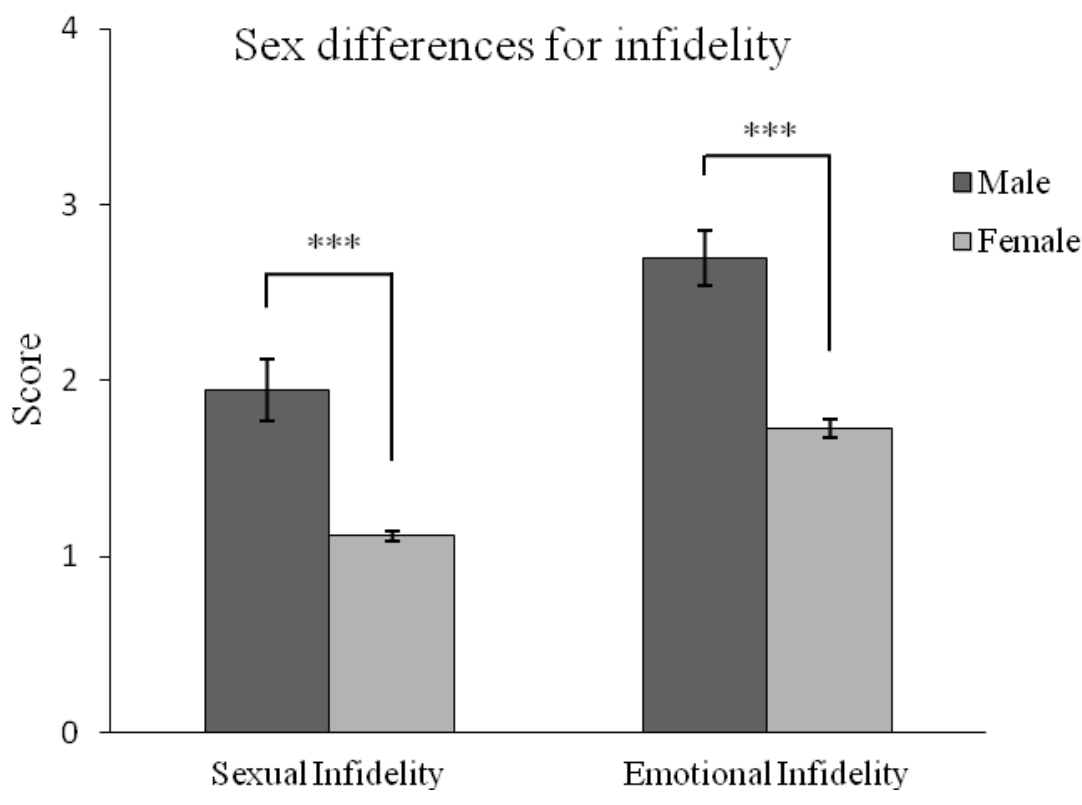




\section{Differences in Relationship Status}

In order to reveal if there are any differences between people who were currently involved in a close relationship and those who were not, a t-test was conducted. Results revealed that individuals who were in a relationship tended to be more emotional promiscuous $t(211.33)=2.45, p<.05, d=.28$, whereas individuals who were not in a relationship tended to be more sexual promiscuous $t(222.96)=-2.66, p<.01, d=-.30$. No differences were found regarding infidelity (neither emotional nor sexual).

\section{Differences in Sexual Orientation}

Unidirectional ANOVAs were performed to examine possible differences in sexual and emotional promiscuity and infidelity depending on the sexual orientation of the participants. Results showed a significant effect of sexual promiscuity, $F(2,356)=23.86, p<.001$. Post-hoc Bonferroni tests showed that there was a significant difference between heterosexuals and homosexuals $\left(p_{\mathrm{s}}<.001\right)$, and between heterosexuals and bisexuals $(\mathrm{p}<.001)$, showing that homosexuals and bisexuals are more sexual promiscuous than heterosexuals. There are no differences between homosexuals and bisexuals regarding sexual promiscuity $(p>.05)$. For emotional promiscuity, no differences were found $F(2,358)=1.35, p>.05$.

In terms of infidelity, differences were found for both domains, that is, for sexual infidelity, $F(2,324)=11.69, p<.001$, and emotional infidelity, $F(2,324)$ $=6.66, p<.01$. Post-hoc Bonferroni tests revealed a significant difference between heterosexuals and bisexuals $\left(p_{\mathrm{s}}<.01\right)$, showing that bisexuals are more sexual and emotional unfaithful than heterosexuals. No differences were found for heterosexuals and homosexuals and homosexuals and bisexuals $\left(p_{\mathrm{s}}>.05\right)$.

\section{Discussion}

Our main goal was to investigate the relationship between sexual and emotional infidelity and promiscuity. To this end, we conducted analysis of the relation between sexual and emotional promiscuity and infidelity. In addition, we explored possible sex differences within these domains.

Regarding the emotional domain, results revealed that there is a positive correlation between emotional promiscuity and emotional infidelity. These findings are consistent with our first hypothesis, and show that individuals that tend to be more emotionally promiscuous also tend to be more emotionally unfaithful.

In terms of the sexual domain, results showed that there is also a positive correlation between sexual promiscuity and sexual infidelity, stating that individuals that tend to be more sexually promiscuous also tend to be more sexually unfaithful. These results support our second hypothesis. 
The results of our study showed that sexual infidelity and emotional infidelity are positively correlated, indicating that individuals that are more sexually unfaithful also tend to be more emotionally unfaithful. These findings are consistent with our third hypothesis.

Regarding promiscuity, our findings suggest that, in the same line as infidelity, sexual and emotional promiscuity are also positively correlated, corroborating our fourth hypothesis. No research relating sexual and emotional promiscuity have been conducted; however we can hypothesize frequent sexual intercourse (sexual promiscuity) rises the possibility of one falling in love with someone (emotional promiscuity) (Jones and Paulhus 2012).

The most popular research on sex differences focused, for promiscuity, on the desire for sexual diversity, whereas for infidelity focused on the jealousy responses based on an evolutionary perspective, as well as which type of unfaithful behaviors are more distressing (Buss et al. 1992, Cramer et al. 2008, Green and Sabini 2006, Penke and Asendorpf 2008). Fewer research has pointed to sex differences between males and females regarding the frequency of unfaithful or promiscuous behaviors. In terms of infidelity, our results suggest that men are more likely to engage in sexual and emotional infidelity than do women, which is consistent with some findings on literature. Seal et al. (1994) studied the willingness to engage in extradyadic behaviors and found that when participants were presented with a hypothetical opportunity, men showed a greater tendency to be unfaithful than women. Moreover, studies also report that, of the people who admitted being unfaithful, women say they engaged more in emotional infidelity than men, whereas men engaged more in sexual infidelity than women (Boekhout et al. 1999, Glass and Wright 1992, Sheppard et al. 1995, Thompson 1984). Possible explanations on sex differences related to infidelity are provided by evolutionary theories (e.g., Cramer et al. 2008). They state that men have difficulty in the matter of forgiving sexual infidelity due to paternal certainty because there is a risk that the male will be investing in another's man offspring, therefore failing to propagate his own genes. Regarding females, emotional infidelity is more harmful than sexual infidelity, because when a male develops emotional involvement with another woman, the risk of losing the resources and long-term commitment increases - thus reducing the probability of bearing a child and disseminate her genes through generations (Wilson et al. 2011).

Nevertheless, there are some studies that did not find sex differences regarding infidelity (e.g., Feldman and Caufmann 1999).

In terms of promiscuity, there are only a few studies that focused on sex differences, showing that men are designed for short-term mating, whereas females for long-term mating (Buss and Schmitt 1993, Kenrick et al. 1990). In an evolutionary perspective, males engage in more unrestricted behaviors due to offspring matters, trying to disseminate as much of their own genes to future generations, while females show more sociosexual restricted behaviors, because once a good mate is selected, the female will secure the mate, given the resources and healthy genes provided to offspring (Treger and Sprecher 2011). Our study, consistently with this literature, found that men tend to engage in more acts of 
sexual promiscuity than do women, whereas no sex differences were found regarding emotional promiscuity, suggesting that both men and women equally engage in emotional promiscuous behaviors. Therefore, our sixth hypothesis was not confirmed. More specifically, men in our sample tended to be more sexual unfaithful and sexual promiscuous than women, but both men and women were equally likely to engage in emotional promiscuous behaviors.

\section{Limitations and Future Research}

First, the average age was approximately 24 years. Therefore, it would be interesting to investigate if the same pattern of results would be obtained with an older sample. Second, because of the correlational nature of the results, no casual associations can be made. Future research could use methodologies which would allow for tests of the hypothesis that there is a causal linkage between promiscuity and infidelity. More specifically, more robust evidence for a causal link between these variables would require a study with a prospective, longitudinal design. Third, we used only one question to assess relationship satisfaction. Future research could use a questionnaire which measures the relationship satisfaction in its different domains (e.g. Perceived Relationship Quality Components, Fletcher et al. 2000, Silva et al. n.d.).

\section{Conclusion}

The data collected from our sample provided a more distinguished insight in the fields of promiscuity and infidelity. Results showed that sexual and emotional promiscuity are related, as well as sexual and emotional infidelity, suggesting that sexual promiscuous people also tend to be emotional promiscuous (and vice-versa) and those who are sexual unfaithful, also tend to be emotional unfaithful (and vice-versa). Additionally, results demonstrated that sexual and emotional promiscuous individuals, also tend to be sexual and emotional unfaithful, being all these domains related to each other.

Sex differences found with the collected data, also provide evidence for the literature, enriching this way and completing studies that research these differences in the field of infidelity and promiscuity. In this study, results indicated that men are more sexual promiscuous than women, yet for emotional promiscuity, no differences were evidenced, stating that both men and women equally engage in emotional promiscuous behaviors. For infidelity, we found that male individuals engage more in sexual and emotional unfaithful behaviors than women.

Results can have implications for several domains. For example, by showing that the promiscuity is related to the infidelity, our study suggests that implementing a comprehensive sex education program focusing in this thematic will likely result in a decrease in promiscuous activities and also in extradyadic behaviors. Generally, the results of our study provide a deeper understanding in 
matters that are relevant on interpersonal relations, until now lacking in literature and offering new possibilities for future research.

\section{References}

Atkins DC, Baucom DH, Jacobson NS (2001) Understanding infidelity: Correlates in a national random sample. Journal of Family Psychology 15: 735-749. doi:10. 1037/0893-3200.15.4.735

Barta WD, Kiene SM (2005) Motivations for infidelity in heterosexual dating couples: The roles of gender, personality differences, and sociosexual orientation. Journal of Social and Personal Relationships 22(3): 339-360. doi:10.1177/0265407505 052440

Boekhout BA, Hendrick SS, Hendrick C (1999) Relationship infidelity: A loss perspective. Journal of Personal \& Interpersonal Loss 4(2): 97-123. doi:10.1080/ 10811449908409721

Brand R J, Markey CM, Mills A, Hodges S D (2007) Sex differences in self-reported infidelity and its correlates. Sex Roles 57: 101-109. doi:10.1007/s11199-0079221-5.

Buss DM, Barnes M (1986) Preferences in human mate selection. Journal of Personality and Social Psychology 50(3): 559.

Buss DM, Larsen RJ, Westen D, Semmelroth J (1992) Sex differences in jealousy: Evolution, physiology, and psychology. Psychological Science 3(4): 251-255.

Buss DM, Schmitt DP (1993) Sexual strategies theory: An evolutionary perspective on human mating. Psychological Review 100(2): 204. doi:10.1037/0033-295X.100. 2.204

Cramer RE, Lipinski RE, Meteer JD, Houska JA (2008) Sex differences in subjective distress to unfaithfulness: Testing competing evolutionary and violation of infidelity expectations hypotheses. The Journal of Social Psychology 148(4): 389-406.

Drigotas SM, Safstrom CA, Gentilia T (1999) An investment model prediction of dating infidelity. Journal of Personality and Social Psychology 77(3): 509. doi:10.1037/ 0022-3514.77.3.509

Feldman SS, Cauffman E (1999) Your cheatin'heart: Attitudes, behaviors, and correlates of sexual betrayal in late adolescents. Journal of Research on Adolescence 9(3): 227-252. doi:10.1207/s15327795jra0903_1

Fletcher GJ, Simpson JA, Thomas G (2000) The measurement of perceived relationship quality components: A confirmatory factor analytic approach. Personality and Social Psychology Bulletin 26(3): 340-354.

Garcia JR, MacKillop J, Aller EL, Merriwether AM, Wilson DS, Lum JK (2010) Associations between dopamine D4 receptor gene variation with both infidelity and sexual promiscuity. Plos One 5(11).

Glass SP, Wright TL (1992) Justifications for extramarital relationships: The association between attitudes, behaviors, and gender. Journal of Sex Research 29(3): 361-387. doi:10.1080/00224499209551654

Green MC, Sabini J (2006) Gender, socioeconomic status, age, and jealousy: Emotional responses to infidelity in a national sample. Emotion 6(2): 330-334. doi:10.1037/ 1528-3542.6.2.330

Jones DN (2011) Emotional promiscuity: consequences for health and well-being (Doctoral Dissertation). Vancouver, Canada: University of British Columbia. 
Jones D N, Paulhus D L (2012) The role of emotional promiscuity in unprotected sex. Psychology \& health 27(9): 1021-1035. doi:10.1080/08870446.2011.647819

Kenrick DT, Sadalla EK, Groth G, Trost MR (1990) Evolution, traits, and the stages of human courtship: Qualifying the parental investment model. Journal of Personality 58(1): 97-116.

Kinsey AC, Pomeroy WP, Martin CE (1949) Sexual behaviour in the human male. Journal of Neuropathology \& Experimental Neurology 8(1): 121-135. doi:10.1002/ ajpa.1330060119

Leeker O, Carlozzi A (2012) Effects of sex, sexual orientation, infidelity expectations, and love on distress related to emotional and sexual infidelity. Journal of Marital and Family Therapy 40: 68-91. doi:10.1111/j.1752-0606.2012.00331.x

Mark KP, Janssen E, Milhausen RR (2011). Infidelity in heterosexual couples: Demographic, interpersonal, and personality-related predictors of extradyadic sex. Archives of Sexual Behavior 40(5): 971-982. doi:10.1007/s10508-011-9771-z

Markey PM, Markey CN (2007) The interpersonal meaning of sexual promiscuity. Journal of Research in Personality 41: 1199-1212. doi:10.1016/j.jrp.2007.02.004

Okafor HC, Duru NE (2010) Sexual promiscuity among female undergraduates in tertiary institutions in imo state: An issue for healthy living. Edo Journal of Counselling 3(1): 100-109.

Penke L, Asendorpf JB (2008) Beyond global sociosexual orientations: A more differentiated look at sociosexuality and its effects on courtship and romantic relationships. Journal of Personality and Social Psychology 95(5): 1113-1135. doi.org/10.1037/0022-3514.95.5.1113

Pinto R, Arantes J (n.d. a). Translation and validation of the sexual and emotional promiscuity scales to Portuguese. Psicothema. [under review]

Pinto R, Arantes, J. (n.d. b). Development of the Sexual and Emotional Infidelity (SEI) scale. Current Psychology. [under review]

Sagarin BJ, Becker DV, Guadagno RE, Nicastle LD, Millevoi A (2003) Sex differences (and similarities) in jealousy: The moderating influence of infidelity experience and sexual orientation of the infidelity. Evolution and Human Behavior 24(1): 1723. doi:10.1016/S1090-5138(02)00106-X

Schmitt DP (2004) The Big Five related to risky sexual behaviour across 10 world regions: Differential personality associations of sexual promiscuity and relationship infidelity. European Journal of Personality 18(4): 301-319.

Seal DW, Agostinelli G, Hannett CA (1994) Extradyadic romantic involvement: Moderating effects of sociosexuality and gender. Sex Roles 31(1): 1-22. doi:10.1007/ BF01560274

Shackelford TK, Buss DM, Bennett K (2002) Forgiveness or breakup: Sex differences in responses to a partner's infidelity. Cognition \& Emotion 16(2): 299-307. doi: 10.1080 /02699930143000202

Sheppard VJ, Nelso ES, Andreoli-Mathie V (1995) Dating relationships and infidelity: Attitudes and behaviors. Journal of Sex \& Marital Therapy 21(3): 202-212. doi:10. 1080/00926239508404399

Silva A, Saraiva M, Albuquerque PB, Arantes J (n.d.). The Influence of Relationship Quality on Attitudes toward and Perceptions of Infidelity. Personal Relationships. [in press]

Simpson JA, Gangestad SW (1991) Individual differences in sociosexuality: Evidence for convergent and discriminant validity. Journal of Personality and Social Psychology 60(6): 870-883. doi:10.1037/0022-3514.60.6.870

Thompson AP (1984) Emotional and sexual components of extramarital relations. Journal of Marriage and the Family 46(1): 35-42. 
Treger S, Sprecher S (2011) The influences of sociosexuality and attachment style on reactions to emotional versus sexual infidelity. Journal of Sex Research 48(5): 413-422. doi:10.1080/00224499.2010.516845

Vangelisti AL, Gerstenberger M (2004) Communication and marital infidelity. In The State of Affairs: Explorations in Infidelity and Commitment, J Duncombe, $\mathrm{K}$ Harrison, G Allan, D Marsden (Eds), 59-78. Mahwah, NJ: Erlbaum.

Vrangalova Z, Ong AD (2014) Who benefits from casual sex? The moderating role of sociosexuality. Social Psychological and Personality Science 5(8): 883-891.

Wilson K, Mattingly BA, Clark EM, Weidler DJ, Bequette AW (2011) The gray area: Exploring attitudes toward infidelity and the development of the Perceptions of Dating Infidelity Scale. The Journal of Social Psychology 151(1): 63-86. doi:10. 1080/00224540903366750. 